\title{
SEI model with varying transmission and mortality rates
}

\author{
Gergely Röst \\ Analysis and Stochastics Research Group, Hungarian Academy of Sciences \\ Bolyai Institute, University of Szeged, Hungary \\ Aradi vértanúk tere 1., H-6725 Szeged
}

\begin{abstract}
An SEI model with distributed delay is proposed where the transmission and the death rates depend on the age of infection. The basic reproduction number $\mathcal{R}_{0}$ is identified as a threshold quantity for the stability of equilibria. If $\mathcal{R}_{0}<$ 1 , then the disease-free equilibrium is globally asymptotically stable and the disease dies out. On the contrary, if $\mathcal{R}_{0}>1$, then a locally asymptotically stable endemic equilibrium appears, and applying a permanence theorem for infinite dimensional systems we obtain that the disease persists in the population.
\end{abstract}

AMS 2000: 92D30, 37C70, 34K10

Keywords: disease model, distributed delay, varying infectivity and death rate

\section{Introduction}

Many compartmental models in mathematical epidemiology assume the homogeneity of the infected class: all individuals in that compartment share the same epidemiological parameters. In reality, as time elapses and the disease develops within the host, its infectivity continuously changes. Disease induced mortality rate may also change during the course of infection. The purpose of this paper is to incorporate these features into an SEI type model. Besides multistage models ${ }^{6,8}$, approaches keeping track of an individual's infection-age have existed ${ }^{1,2,5,7}$ to capture this variability. However, the model we formulate in this paper differs from the previous ones since it can be transformed into a system of differential equation with distributed delays, which is easier to deal with than integro-differential or Volterra-type models.

The paper is organized as follows. In section 2 , taking into account the age of infection as a parameter, and allowing varying infectivity and death rates, we formulate an SEI model with distributed and constant delays. We identify the basic reproduction number $\mathcal{R}_{0}$ in terms of the model parame- 
terers as a threshold quantity in section 3 . If $\mathcal{R}_{0}<1$, the disease dies out and all solutions converge to the disease free equilibrium. In section 4 we show that a stable endemic equilibrium appears if $\mathcal{R}_{0}>1$. In section 5 we prove that the disease is endemic in the sense of permanence whenever $\mathcal{R}_{0}>1$.

\section{Derivation of the model}

We divide a given population into the following categories: susceptibles (those who are capable of contracting the disease); exposed (those who are infected but not yet infectious); infectives (those who are infected and capable of transmitting the disease). Denote the number of individuals at time $t$ in these classes by $S(t), E(t), I(t)$, respectively. Let $i(t, a)$ represent the density of infected individuals with respect to the age of infection $a$ at the current time $t$, where $a \leq \tau$, then $I(t)=\int_{0}^{\tau} i(t, a) d a$. We introduce the function $0 \leq \beta(a) \leq \beta$ to express the infectivity according to the age of infection $a$. In what follows, $\Lambda$ denotes the constant recruitment rate, $\beta$ is the maximal transmission rate, $\Delta$ is the natural death rate, $\delta(a) \geq 0$ is the disease-induced death rate which depends on the age of infection as well, $1 / \mu$ is the average incubation period. At age $\tau$ of infection, we remove all remaining individuals from the class $I$ who has survived. Thus, $\tau>0$ represents the maximal duration of the infectious period. All the constants above are assumed to be positive. Then, using bilinear incidence in the force of infection corrected by the infectivity factor due to the age of infection, we arrive at the SEI type model

$$
\begin{aligned}
& \frac{d S(t)}{d t}=\Lambda-S(t) \int_{0}^{\tau} \beta(a) i(t, a) d a-\Delta S(t) \\
& \frac{d E(t)}{d t}=S(t) \int_{0}^{\tau} \beta(a) i(t, a) d a-(\mu+\Delta) E(t), \\
& \frac{d I(t)}{d t}=\mu E(t)-\Delta I(t)-\int_{0}^{\tau} \delta(a) i(t, a) d a-i(t, \tau)
\end{aligned}
$$

The time evolution of the density $i(t, a)$ is given by

$$
\left(\frac{\partial}{\partial t}+\frac{\partial}{\partial a}\right) i(t, a)=-(\Delta+\delta(a)) i(t, a)
$$

subject to the boundary condition

$$
i(t, 0)=\mu E(t) .
$$


Solving (1) leads to

$$
i(t, a)=i(t-a, 0) e^{-\left(\Delta a+\int_{0}^{a} \delta(u) d u\right)}=\mu E(t-a) e^{-\left(\Delta a+\int_{0}^{a} \delta(u) d u\right)},
$$

and we obtain the following deterministic model of delay differential equations with distributed and constant delays:

$$
\begin{aligned}
\frac{d S(t)}{d t}= & \Lambda-S(t) \int_{0}^{\tau} \beta(a) \mu E(t-a) e^{-\left(\Delta a+\int_{0}^{a} \delta(u) d u\right)} d a-\Delta S(t) \\
\frac{d E(t)}{d t}= & S(t) \int_{0}^{\tau} \beta(a) \mu E(t-a) e^{-\left(\Delta a+\int_{0}^{a} \delta(u) d u\right)} d a-(\mu+\Delta) E(t) \\
\frac{d I(t)}{d t}= & \mu E(t)-\Delta I(t)-\int_{0}^{\tau} \delta(a) \mu E(t-a) d a \\
& -e^{-\left(\Delta \tau+\int_{0}^{\tau} \delta(u) d u\right)} \mu E(t-\tau) .
\end{aligned}
$$

From (2) we can express $I(t)$ as a function of a solution $E(t)$ :

$$
I(t)=\mu \int_{0}^{\tau} E(t-a) e^{-\left(\Delta a+\int_{0}^{a} \delta(u) d u\right)} d a .
$$

All the information (boundedness, convergence, etc.) for $I(t)$ can be obtained from the $E$-component of the solution, and equation (5) decouples. Therefore it is sufficient to restrict our attention to the system (3-4), and we do this in the sequel. Clearly the state of system (3-4) at time $t$ is specified by $S(t) \in \mathbb{R}$ and $E_{t} \in C([-\tau, 0], \mathbb{R})$, the space of continuous functions on the interval $[-\tau, 0]$. It is straightforward to see that solutions of $(3-4)$ preserve non-negativity.

Proposition 2.1. The system (3-4) is point dissipative; that is there exists an $M>0$ such that for any non-negative solution of (3-4), there exists a $T>0$ such that $S(t) \leq M$ and $E(t) \leq M$ for all $t \geq T$.

Proof. Consider an arbitrary nonnegative solution. For $W(t)=S(t)+$ $E(t)$, we have

$$
\frac{d W(t)}{d t}=\Lambda-\Delta W(t)-\mu E(t) \leq \Lambda-\Delta W(t) .
$$

Since any nonnegative solution of $w^{\prime}(t)=\Lambda-\Delta w(t)$ satisfies

$$
\lim _{t \rightarrow \infty} w(t)=\Lambda / \Delta
$$

by a standard comparison argument we obtain

$$
\limsup _{t \geq 0} W(t) \leq \frac{\Lambda}{\Delta}
$$


We conclude that for any $\varepsilon>0$, there is a $T>0$ such that the nonnegative solution of (3-4) satisfies

$$
S(t) \leq \frac{\Lambda}{\Delta}+\varepsilon, \quad E(t) \leq \frac{\Lambda}{\Delta}+\varepsilon
$$

whenever $t \geq T$. Consequently, we can choose any $M>\frac{\Lambda}{\Delta}$. Additionally, we obtain the a-priori estimate

$$
S(t), E(t) \leq W(t) \leq \Lambda / \Delta+\exp (-\Delta t)(S(0)+E(0)-\Lambda / \Delta)
$$

\section{Basic reproduction number and the global stability of the disease-free equilibrium}

Clearly our model has a disease-free equilibrium $P_{0}=\left(S_{0}, 0\right)$ where $S_{0}=\Lambda / \Delta$. To find the basic reproduction number $\mathcal{R}_{0}$, we introduce a single exposed individual into a totally susceptible population in the disease-free equilibrium at $t=0$. The probability of the presence of this individual in the $E$-class after time $t$ is given by $e^{-(\mu+\Delta) t}$, so the expected number of generated secondary infections can be calculated by

$$
\mathcal{R}_{0}=S_{0} \int_{0}^{\infty} \int_{0}^{\tau} \beta(a) \mu e^{-\left(\Delta a+\int_{0}^{a} \delta(u) d u\right)} e^{-(\mu+\Delta) t} d a d t
$$

which, after interchanging the integrals, reduces to

$$
\mathcal{R}_{0}=\frac{S_{0} \mu}{\mu+\Delta} \int_{0}^{\tau} \beta(a) e^{-\left(\Delta a+\int_{0}^{a} \delta(u) d u\right)} d a .
$$

Next we show that $\mathcal{R}_{0}$ determines the stability of the disease-free equilibrium and the disease dies out when $\mathcal{R}_{0}<1$.

Theorem 3.1. The disease free equilibrium is globally asymptotically stable if $\mathcal{R}_{0}<1$, and unstable if $\mathcal{R}_{0}>1$.

Proof. For any $\varepsilon>0$, we define

$$
\mathcal{R}_{\varepsilon}=\frac{\mu}{\mu+\Delta}\left(\frac{\Lambda}{\Delta}+\varepsilon\right) \int_{0}^{\tau} \beta(a) e^{-\left(\Delta a+\int_{0}^{a} \delta(u) d u\right)} d a .
$$

Then $\lim _{\varepsilon \rightarrow 0} \mathcal{R}_{\varepsilon}=\mathcal{R}_{0}$ and $\mathcal{R}_{\varepsilon}<1$ if $\mathcal{R}_{0}<1$ and $\varepsilon$ is sufficiently small. In Proposition 1. we have shown that for any $\varepsilon>0$ there is a $T>0$ such that $S(t) \leq \frac{\Lambda}{\Delta}+\varepsilon$ whenever $t>T$. Thus, without loss of generality, we can suppose that $S(t) \leq \frac{\Lambda}{\Delta}+\varepsilon$ for all $t \geq 0$. This yields that the exposed population $E(t)$ is bounded above by the solutions of the linear equation

$$
\frac{d E(t)}{d t}=\left(\frac{\Lambda}{\Delta}+\varepsilon\right) \int_{0}^{\tau} \beta(a) \mu E(t-a) e^{-\left(\Delta a+\int_{0}^{a} \delta(u) d u\right)} d a-(\mu+\Delta) E(t) .
$$


Now we show that the characteristic roots of this linear equation have negative real parts and the global stability of the disease-free equilibrium follows from the standard comparison argument. Using the exponential Ansatz $e^{\lambda t}$, we arrive at the characteristic function

$$
h(\lambda)=\left(\frac{\Lambda}{\Delta}+\varepsilon\right) \mu \int_{0}^{\tau} \beta(a) e^{-\left(\lambda a+\Delta a+\int_{0}^{a} \delta(u) d u\right)} d a-(\lambda+\mu+\Delta) .
$$

We check that each characteristic root has negative real part. Suppose that $\lambda=x+i y$ is a root of $h(\lambda)$ with $x>0$. Then $\left|e^{-\lambda a}\right|<1$ for any $a>0$, and

$$
\begin{aligned}
1 & =\left|\frac{\left(\frac{\Lambda}{\Delta}+\varepsilon\right) \mu}{\lambda+\mu+\Delta} \int_{0}^{\tau} \beta(a) e^{-\left(-\lambda a+\Delta a+\int_{0}^{a} \delta(u) d u\right)} d a\right| \\
& \leq \frac{\left(\frac{\Lambda}{\Delta}+\varepsilon\right) \mu}{|\lambda+\mu+\Delta|} \int_{0}^{\tau} \beta(a)\left|e^{-\lambda a}\right| e^{-\left(\Delta a+\int_{0}^{a} \delta(u) d u\right)} d a<\mathcal{R}_{\varepsilon},
\end{aligned}
$$

which is a contradiction. Therefore, if $\mathcal{R}_{0}<1$, then all roots have negative real part, thus $\lim _{t \rightarrow \infty} E(t)=0$ and all solutions converge to the disease free equilibrium.

If $\mathcal{R}_{0}>1$, then the linearization about the disease free equilibrium gives for (4) that

$$
\frac{d E(t)}{d t}=S_{0} \mu \int_{0}^{\tau} \beta(a) E(t-a) e^{-\left(\Delta a+\int_{0}^{a} \delta(u) d u\right)} d a-(\mu+\Delta) E(t),
$$

which leads to the characteristic function

$$
\hat{h}(\lambda)=S_{0} \mu \int_{0}^{\tau} \beta(a) e^{-\left(\lambda a+\Delta a+\int_{0}^{a} \delta(u) d u\right)} d a-(\lambda+\mu+\Delta) .
$$

Clearly, $\hat{h}(\lambda)$ is a monotone decreasing continuous function for nonnegative real $\lambda$ and $\hat{h}(\infty)=-\infty$. We have

$$
\hat{h}(0)=S_{0} \mu \int_{0}^{\tau} \beta(a) e^{-\left(\Delta a+\int_{0}^{a} \delta(u) d u\right)} d a-(\mu+\Delta)=(\mu+\Delta)\left(\mathcal{R}_{0}-1\right) .
$$

If $\mathcal{R}_{0}>1$, then there exists a positive real root of $\hat{h}(\lambda)$, and the disease-free equilibrium is unstable.

\section{The endemic equilibrium}

Theorem 4.1. An endemic equilibrium exists if and only if $\mathcal{R}_{0}>1$. Moreover, the endemic equilibrium, if exists, is unique and locally asymptotically stable. 
Proof. An endemic equilibrium $P^{*}=\left(S^{*}, E^{*}\right)$ must satisfy the algebraic equations

$$
\begin{aligned}
\Delta S^{*} & =\Lambda-S^{*} \mu \int_{0}^{\tau} \beta(a) E^{*} e^{-\left(\Delta a+\int_{0}^{a} \delta(u) d u\right)} d a, \\
(\mu+\Delta) E^{*} & =S^{*} \mu \int_{0}^{\tau} \beta(a) E^{*} e^{-\left(\Delta a+\int_{0}^{a} \delta(u) d u\right)} d a .
\end{aligned}
$$

Since $E^{*} \neq 0,(10)$ yields

$$
S_{0} / S^{*}=\mathcal{R}_{0}, \quad \text { or } \quad S^{*}=\frac{\Lambda}{\mathcal{R}_{0} \Delta} .
$$

Simple calculations on (9) show that

$$
\frac{\Lambda}{\mathcal{R}_{0}}=\Lambda-(\Delta+\mu) E^{*}
$$

that is

$$
E^{*}=\frac{\Lambda}{\Delta+\mu}\left(1-\frac{1}{\mathcal{R}_{0}}\right) .
$$

So, we conclude that $E^{*}>0$ if and only if $\mathcal{R}_{0}>1$.

Next we show the local asymptotic stability of the endemic equilibrium. Introducing the new variables $s(t)=S(t)-S^{*}, f(t)=E(t)-E^{*}$, we obtain the linearized system about the endemic equilibrium $P^{*}=\left(S^{*}, E^{*}\right)$

$$
\begin{aligned}
& \frac{d s(t)}{d t}=-\int_{0}^{\tau}\left(s(t) E^{*}+S^{*} e(t-a)\right) \mu \beta(a) e^{-\left(\Delta a+\int_{0}^{a} \delta(u) d u\right)} d a-\Delta s(t), \\
& \frac{d e(t)}{d t}=\int_{0}^{\tau}\left(s(t) E^{*}+S^{*} e(t-a)\right) \mu \beta(a) e^{-\left(\Delta a+\int_{0}^{a} \delta(u) d u\right)} d a-(\mu+\Delta) e(t) .
\end{aligned}
$$

Noticing

$$
\mu \int_{0}^{\tau} \beta(a) E^{*} e^{-\left(\Delta a+\int_{0}^{a} \delta(u) d u\right)} d a=\mathcal{R}_{0}-1
$$

we have

$$
\begin{aligned}
& \frac{d s(t)}{d t}=-s(t)\left(\mathcal{R}_{0}-1\right)-\frac{\Lambda \mu}{\mathcal{R}_{0} \Delta} \int_{0}^{\tau} \beta(a) e(t-a) e^{-\left(\Delta a+\int_{0}^{a} \delta(u) d u\right)} d a-\Delta s(t), \\
& \frac{d e(t)}{d t}=s(t)\left(\mathcal{R}_{0}-1\right)+\frac{\Lambda \mu}{\mathcal{R}_{0} \Delta} \int_{0}^{\tau} \beta(a) e(t-a) e^{-\left(\Delta a+\int_{0}^{a} \delta(u) d u\right)} d a-(\mu+\Delta) e(t) .
\end{aligned}
$$

Using the exponential Ansatz $e^{\lambda t}\left(s_{0}, e_{0}\right)$, we have

$$
(\lambda+\Delta) s_{0}=-(\lambda+\mu+\Delta) e_{0}
$$


and

$$
\left(\mathcal{R}_{0}-1+\Delta+\lambda\right) s_{0}=-\left(\frac{\Lambda \mu}{\mathcal{R}_{0} \Delta} \int_{0}^{\tau} \beta(a) e^{-\lambda a} e^{-\left(\Delta a+\int_{0}^{a} \delta(u) d u\right)} d a\right) e_{0},
$$

thus we obtain the characteristic equation

$$
(\lambda+\mu+\Delta)\left(\mathcal{R}_{0}-1+\Delta+\lambda\right)=(\lambda+\Delta)\left(\frac{\Lambda \mu}{\mathcal{R}_{0} \Delta} \int_{0}^{\tau} \beta(a) e^{-\lambda a} e^{-\left(\Delta a+\int_{0}^{a} \delta(u) d u\right)} d a\right) .
$$

Suppose that $\lambda=x+i y$ is a root and $x \geq 0$, that implies $\left|e^{-\lambda a}\right| \leq 1$ for any $a \geq 0$. Now the inequalities

$$
\left|\frac{\Lambda \mu}{\mathcal{R}_{0} \Delta} \int_{0}^{\tau} \beta(a) e^{-\lambda a} e^{-\left(\Delta a+\int_{0}^{a} \delta(u) d u\right)} d a\right| \leq \mu+\Delta \leq|\lambda+\mu+\Delta|
$$

and

$$
|\Delta+\lambda|<\left|\mathcal{R}_{0}-1+\Delta+\lambda\right|
$$

follow, contradicting to (12). Therefore, every root has negative real part and the endemic equilibrium is locally asymptotically stable if $\mathcal{R}_{0}>1$.

\section{Persistence}

Denote by $T(t): X \rightarrow X, t \geq 0$ the family of solution operators corresponding to (3-4), where $X=R_{0}^{+} \times C_{0}^{+}$. Here $C_{0}^{+}$denotes the set of nonnegative continuous functions on the interval $[-\tau, 0]$. The $\omega$-limit set $\omega(x)$ of $x$ consists of $y \in X$ such that there is a sequence $t_{n} \rightarrow \infty$ as $n \rightarrow \infty$ with $T\left(t_{n}\right) x \rightarrow y$ as $n \rightarrow \infty$. We shall apply the following permanence theorem of Hale \& Waltman ${ }^{3}$, in the spirit of Röst \& $\mathrm{Wu}^{4}$.

Theorem. Suppose that we have the following:

(i) $X^{0}$ is open and dense in $X$ with $X^{0} \cup X_{0}=X$ and $X^{0} \cap X_{0}=\emptyset$;

(ii) the solution operators $T(t)$ satisfy

$$
T(t): X^{0} \rightarrow X^{0}, \quad T(t): X_{0} \rightarrow X_{0}
$$

(iii) $T(t)$ is point dissipative in $X$;

(iv) there is a $t_{0} \geq 0$ such that $T(t)$ is compact for all $t \geq t_{0}$;

(v) $\mathcal{A}=\bigcup_{x \in A_{b}} \omega(x)$ is isolated and has an acyclic covering $N$, where

$A_{b}$ is the global attractor of $T(t)$ restricted to $X_{0}$ and $N=\cup_{i=1}^{k} N_{i}$;

(vi) for each $N_{i} \in N$,

$$
W^{s}\left(N_{i}\right) \cap X^{0}=\emptyset,
$$

where $W^{s}$ refers to the stable set. 
Then $T(t)$ is a uniform repeller with respect to $X^{0}$, i.e. there is an $\eta>0$ such that for any $x \in X^{0}, \liminf _{t \rightarrow \infty} d\left(T(t) x, X_{0}\right) \geq \eta$.

Theorem 5.1. If $\mathcal{R}_{0}>1$, then the disease is endemic; more precisely, there exists an $\eta>0$ such that

$$
\liminf _{t \rightarrow \infty} E(t) \geq \eta
$$

Proof. Let

$$
\begin{gathered}
X^{0}=\{(S, \phi) \in X: \phi(\theta)>0 \text { for some } \theta<0\} \\
X_{0}=\{(S, \phi) \in X: \phi(\theta)=0 \text { for all } \theta \leq 0,\} .
\end{gathered}
$$

We check all the conditions of the permanence theorem. It is straightforward to see that (i) and (ii) are satisfied. The point dissipativity has been proved in Proposition 1, so we have (iii). Applying the Arzela-Ascoli theorem we obtain (iv) with $t_{0}=\tau$.

Regarding (v), clearly $\mathcal{A}=\left\{P_{0}\right\}$ (now $P_{0}=(\Lambda / \Delta, 0) \in X$ ) and isolated. Hence the covering is simply $N=\left\{P_{0}\right\}$, which is acyclic (there is no orbit which connects $P_{0}$ to itself in $\left.X_{0}\right)$.

It remains to show that $W^{s}\left(P_{0}\right) \cap X^{0}=\emptyset$. Suppose the contrary, that is there exists a solution in $X^{0}$ such that

$$
\lim _{t \rightarrow \infty} S(t)=S_{0}, \quad \lim _{t \rightarrow \infty} E(t)=0 .
$$

Since $\mathcal{R}_{0}>1$, there exists an $\varepsilon>0$ such that

$$
\left(S_{0}-\varepsilon\right) \mu \int_{0}^{\tau} \beta(a) e^{-\left(\Delta a+\int_{0}^{a} \delta(u) d u\right)} d a>\mu+\Delta .
$$

There exists a $t_{0}$ such that for $t \geq t_{0}, S(t)>S_{0}-\varepsilon$ and hence

$$
E^{\prime}(t) \geq\left(S_{0}-\varepsilon\right) \mu \int_{0}^{\tau} \beta(a) E(t-a) e^{-\left(\Delta a+\int_{0}^{a} \delta(u) d u\right)} d a-(\mu+\Delta) E(t) .
$$

If $E(t) \rightarrow 0$, as $t \rightarrow \infty$, then by a standard comparison argument and the nonnegativity, the solution $n(t)$ of

$$
n^{\prime}(t)=\left(S_{0}-\varepsilon\right) \mu \int_{0}^{\tau} \beta(a) n(t-a) e^{-\left(\Delta a+\int_{0}^{a} \delta(u) d u\right)} d a-(\mu+\Delta) n(t)
$$

with initial data $n_{0}=E_{0}$, has to converge to 0 as well. By the mean value theorem for integrals we have that for any $t$ there is a $\xi_{t}$ such that

$$
\int_{0}^{\tau} \beta(a) n(t-a) e^{-\left(\Delta a+\int_{0}^{a} \delta(u) d u\right)} d a=n\left(\xi_{t}\right) \int_{0}^{\tau} \beta(a) e^{-\left(\Delta a+\int_{0}^{a} \delta(u) d u\right)} d a
$$


and $t-\tau \leq \xi_{t} \leq t$. Define

$$
V(t):=n(t)+(\mu+\Delta) \int_{\xi_{t}}^{t} n(s) d s .
$$

Differentiating with respect to time gives

$$
\frac{d V}{d t}=\left(\beta\left(S_{0}-\varepsilon\right) \int_{0}^{\tau} k(a) \mu e^{-\left(\Delta a+\int_{0}^{a} \delta(u) d u\right)} d a-(\mu+\Delta)\right) n\left(\xi_{t}\right) \geq 0 .
$$

Therefore, $V(t)$ goes to infinity or approaches a positive limit as $t \rightarrow \infty$. On the other hand, by the definition of $V$, $\lim _{t \rightarrow \infty} n(t)=0$ implies $\lim _{t \rightarrow \infty} V(t)=0$, a contradiction. Thus $W^{s}\left(P_{0}\right) \cap X^{0}=\emptyset$ and we can apply Theorem 4.2 of Hale \& Waltman ${ }^{3}$ to obtain that for some $\eta>0$,

$$
\liminf _{t \rightarrow \infty} E(t)>\eta
$$

Though our calculations has been done for the reduced system (3-4), and we are interested in the dynamics of the infectious class, we easily obtain that for $\mathcal{R}_{0}<1, E(t) \rightarrow 0$ implies $I(t) \rightarrow 0$, and for $\mathcal{R}_{0}>1$ from (2) we have the endemic equilibrium

$$
I^{*}=\frac{\Lambda \mu}{\Delta+\mu}\left(1-\frac{1}{\mathcal{R}_{0}}\right)\left(\int_{0}^{\tau} e^{-\left(\Delta a+\int_{0}^{a} \delta(u) d u\right)} d a\right),
$$

furthermore, from (2) we obtain

$$
\liminf _{t \rightarrow \infty} I(t)>\mu \eta \int_{0}^{\tau} e^{-\left(\Delta a+\int_{0}^{a} \delta(u) d u\right)} d a>0 .
$$

Hence, applying the permanence theorem above, we obtain that the disease will always be present in the population when $\mathcal{R}_{0}>1$.

\section{Acknowledgements}

The author acknowledges the support of the Hungarian Scientific Research Fund, grant OTKA K75517, the Bolyai Research Scholarship of the Hungarian Academy of Sciences, and the TÁMOP-4.2.2/08/1/2008-0008 program of the Hungarian National Development Agency.

\section{References}

1. C. Castillo-Chavez, K. Cooke, W. Huang \& S. A. Levin. On the role of long incubation periods in the dynamics of acquired immunodeficiency syndrome (AIDS). I. Single population models J. Math. Biol. 27(1989), 373-398.

2. H. R. Thieme \& C. Castillo-Chavez. How may infection-age-dependent infectivity affect the dynamics of HIV/AIDS? SIAM J. Appl. Math. 53(1993), 1447-1479. 
3. J. K. Hale \& P. Waltman. Persistence in infinite-dimensional systems. SIAM J. Math. Anal. 20(1989), 388-395.

4. G. Röst \& J. Wu SEIR epidemiological model with varying infectivity and infinite delay Math. Biosci. Eng. 5:(2)(2008) 389-402

5. Y. Zhou, Y. Shao, Y. Ruan, J. Xu, Z. Ma, C. Mei \& J. Wu Modeling and prediction of HIV in China: transmission rates structured by infection ages. Math. Biosci. Eng. 5:(2)(2008) 403-418

6. A. B. Gumel, C. C. McCluskey \& P. van den Driessche Mathematical study of a staged-progression HIV model with imperfect vaccine, Bull. Math. Biol. 68:(8)(2006) 2105-2128

7. H. Inaba Endemic threshold results in an age-duration-structured population model for HIV infection Math. Biosci. 201:(1-2)(2006) 15-47

8. B. D. Corbett, S. M. Moghadas \& A. B. Gumel, Subthreshold domain of bistable equilibria for a model of HIV epidemiology, Int. J. Math. Math. Sci. 58(2003) 3679-3698 